\title{
Current Research Progress of Ceramic Biomaterials in Japan
}

\author{
Kimihiro Yamashita ${ }^{\mathrm{a}}$ and Masayuki Okazaki ${ }^{\mathrm{b}}$ \\ ${ }^{a}$ Tokyo Medical \& Dental University, Japan \\ ${ }^{\mathrm{b}}$ Hiroshima University, Jikei Institute, Japan
}

The R\&D of ceramic biomaterials has extensively progressed in Japan since commercial production of calcium phosphates of hydroxyapatite and $\beta$-tricalcium phosphate, A-W glass, chemicals- and SBF (simulated body fluid)-treated metals, and yttria-stabilized zirconia for dental and medical applications. The centers of R\&D excellence in Japan consist of universities and national research institutes in addition to industrial companies. The public organizations are listed below for reference.

Hokkaido and Tohoku districts

Hokkaido U, Tohoku U, Yamagata U, Health Sciences U of Hokkaido, Hokkaido Research Organization

Kanto district

Tokyo Institute of Technology, Tokyo Medical and Dental U, Meiji U, Chiba Institute of Technology, Nihon U, Yamanashi U, National Institute of Advanced Industrial Science and Technology, National Institute for Materials Science

Tokai district

Nagoya U, Nagoya Institute of Technology, Japan Fine Ceramics Center

Kansai and Chugoku districts

Kyoto U, Osaka City U, Osaka Prefectural U, Kinki U, Okayama U, Hiroshima U

Kyushu and Shikoku districts

Kyushu U, Kyushu Institute of Technology

We, the members of Japanese strategic group for future ceramic biomaterials $R \& D$, have discussed on the publication of our current research results for the worldwide R\&D of ceramic biomaterials. In answer to our request, 5 leading organizations were willing to contribute to our action. The issue covers from syntheses of novel biomaterials of carbonated OCP (named as OCPC) and phosphate glass through the fundamental studies of ion releasing biological effects, protein adsorption mechanisms, and biological evaluation of hydroxyapatite to discovery of the nature of human bones. Especially, one of the guest 
editors (MO) presents his scientific and philosophical review on the fundamental structural issue. We believe that these 8 papers will bring great profits to the readers.

Lastly, we deeply express our gratitude to Prof. A.T. Yokobori, Jr., Editor-in-Chief, for his continuous encouragement to our publication.

Guest Editors

Kimihiro Yamashita

Masayuki Okazaki
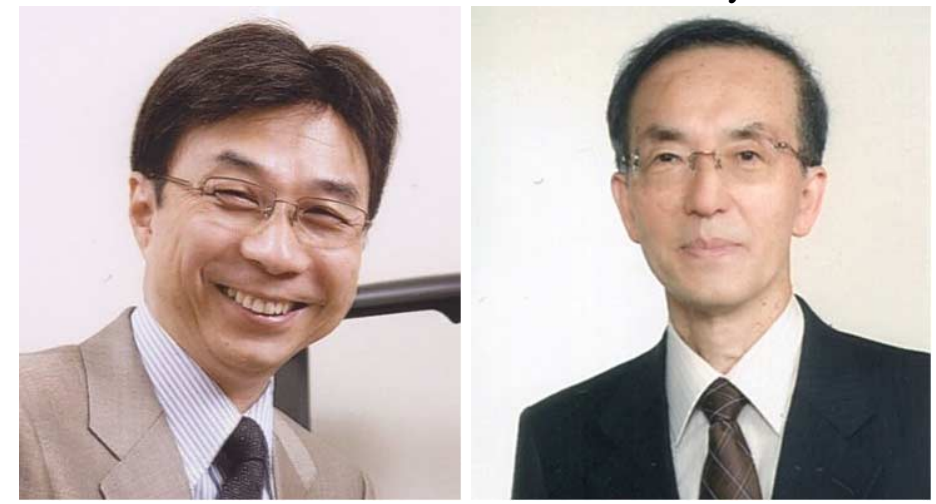